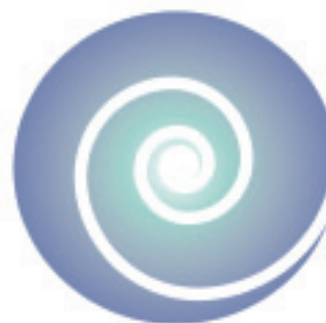

\title{
Clinical, Biomechanical, and Physiological Translational Interpretations of Human Resting Myofascial Tone or Tension
}

Alfonse T. Masi, MD, DrPH, ${ }^{1}$ Kalyani Nair, PhD, ${ }^{2}$ Tyler Evans, BS, ${ }^{3}$ Yousef Ghandour, PT, MOMT, FAAOMPT 4

${ }^{I}$ Departments of Medicine and Epidemiology, University of Illinois, College of Medicine at Peoria, Peoria, IL; ${ }^{2}$ Department of Mechanical Engineering, Bradley University, Peoria, IL; ${ }^{3}$ University of Illinois, College of Medicine at Peoria, Peoria, IL; and ${ }^{4}$ Ola Grimsby Institute, Santee, CA, USA

Background: Myofascial tissues generate integrated webs and networks of passive and active tensional forces that provide stabilizing support and that control movement in the body. Passive [central nervous system (CNS)-independent] resting myofascial tension is present in the body and provides a low-level stabilizing component to help maintain balanced postures. This property was recently called "human resting myofascial tone" (HRMT). The HRMT model evolved from electromyography (EMG) research in the 1950s that showed lumbar muscles usually to be EMG-silent in relaxed gravity-neutral upright postures.

Methods: Biomechanical, clinical, and physiological studies were reviewed to interpret the passive stiffness properties of HRMT that help to stabilize various relaxed functions such as quiet balanced standing. Biomechanical analyses and experimental studies of the lumbar multifidus were reviewed to interpret its passive stiffness properties. The lumbar multifidus was illustrated as the major core stabilizing muscle of the spine, serving an important passive biomechanical role in the body.

Results: Research into muscle physiology suggests that passive resting tension (CNS-independent) is generated in sarcomeres by the molecular elasticity of low-level cycling cross-bridges between the actomyosin filaments. In turn, tension is complexly transmitted to intimately enveloping fascial matrix fibrils and other molecular elements in connective tissue, which, collectively, constitute the myofascial unit. Postural myofascial tonus varies with age and sex. Also, individuals in the population are proposed to vary in a polymorphism of postural HRMT. A few people are expected to have outlier degrees of innate postural hypotonicity or hypertonicity. Such biomechanical variations likely predispose to greater risk of related musculoskeletal disorders, a situation that deserves greater attention in clinical practice and research. Axial myofascial hypertonicity was hypothesized to predispose to ankylosing spondylitis. This often-progressive deforming condition of vertebrae and sacroiliac joints is characterized by stiffness features and particular localization of bony lesions at entheseal sites. Such unique features imply concentrations and transmissions of excessive force, leading to tissue micro-injury and maladaptive repair reactions.

Conclusions: The HRMT model is now expanded and translated for clinical relevance to therapists. Its passive role in helping to maintain balanced postures is supported by biomechanical principles of myofascial elasticity, tension, stress, stiffness, and tensegrity. Further research is needed to determine the molecular basis of HRMT in sarcomeres, the transmission of tension by the enveloping fascial elements, and the means by which the myofascia helps to maintain efficient passive postural balance in the body. Significant deficiencies or excesses of postural HRMT may predispose to symptomatic or pathologic musculoskeletal disorders whose mechanisms are currently unexplained.

KEYWORDS: Resting muscle, tone, tension, stiffness, passive, elasticity, fascia, myofascia, crossbridge theory, sarcomere

\section{INTRODUCTION}

The topic of passive resting ("static") myofascial tonicity (or tension) has long been controversial, as recently reviewed ${ }^{(1-3)}$. Nevertheless, a multidisciplinary perspective supports the role of human resting myofascial tone (HRMT) in helping to stabilize the lumbar spine during balanced postures. This passive (static) tone has elastic properties (that is, without definite hysteresis behavior), which differ from the more inclusive viscoelasticity quality, which occurs in movement conditions ${ }^{(3)}$ (Table 1). Anatomical, biomechanical, and functional implications of HRMT are reviewed here as they relate to medical and manual therapy interests. The broad scope of this review necessitates simplification of the proposed molecular basis for HRMT and allows only for a description of 
TABLE 1. Biomechanical Terms Relevant to Myofascial Tone (Tension)

Stress $(\sigma)$

Strain $(\varepsilon)$

Elasticity

Viscoelasticity

Active tone (tension)

Passive tone

Resting (static) myofascial tone

Dynamic or kinetic (nonstatic) myofascial tone

Modulus of elasticity $(E)$

Stiffness $(k)$

Stable system

Structural stability

Plasticity

Viscosity

Thixotropy

Resonant frequency of a vibrating body
The force (tension) per unit area over which the force acts ( $\sigma=F /$ area); in muscle, it is normalized by the dimension of physiological cross-sectional area.

The amount of deformation that results from an applied force, expressed as the proportionate change in length $(\Delta L)$ from the original length $L_{\mathrm{o}}\left(\varepsilon=\Delta L / L_{\mathrm{o}}\right)$.

The property of a material to return to its original form or shape after a deforming force is removed. Elasticity refers to both the static (linear) and dynamic (viscoelastic) relations between stress and strain.

The property of being both elastic and viscous

The central nervous system-dependent force generated by a muscle when stimulated and contracting, either in the static or the kinetic state.

An intrinsic tensile central nervous system-independent force of myofascial tissue when not stimulated or actively contracting, which occurs in the static (linear) and the kinetic (viscoelastic) conditions.

The elastic component of passive tone, within the linear range of the tension versus lengthening curve (spring-like).

The viscoelastic component of passive tone within the nonlinear range of imposed extension, in which the resistive tension varies with the rate of lengthening.

An intrinsic material property expressed as the ratio of an applied stress ( $\sigma=F /$ area) to the resulting $\operatorname{strain}\left(\varepsilon=\Delta L / L_{\mathrm{o}}\right)$, as characterized by "stress over strain" in Hooke's Law $(E=\sigma / \varepsilon)$. Depending upon the types of loading, modulus of elasticity may be reported as

- compressive modulus of elasticity ( $E$ in compression).

- flexural (bending) modulus of elasticity ( $E$ in flexure)

- shear (surface sliding) modulus of elasticity ( $E$ in shear).

- tensile modulus of elasticity ( $E$ in tension).

- torsional (twisting) modulus of elasticity ( $E$ in torsion).

A material's resistance to deformation $(\Delta L)$ by an applied compressive or tensile force $(F)$, defined as $k=$ $F / \Delta L$.

A system having equilibrium of its balanced forces and moments, which permits restoration responses to small perturbations, such as minor elastic oscillations or vibrations.

A simplified concept exists if a small displacement or perturbation produces a restoring force in the body, such as an elastic response.

The material property of being plastic (from the Greek plastikos, relating to molding) or capable of being formed or molded into a permanently deformed shape by pressure or heat.

The resistance to flow of a nonsolid substance that is measured as shear force required to obtain a rate of deformation, which is time-dependent.

The property exhibited by certain gels, such as muscle, of becoming more fluid when internally agitated or moved (kinetic), and returning to a more viscous state after standing (static).

The frequency at which the amplitude of a response to an applied force is maximum, and is determined, in part, by the stiffness $(k)$ of the system. the more accepted anatomical relations. Also, the essential role of fascial elements in force transmission ${ }^{(4)}$ is covered to a lesser degree than its proposed generation in sarcomeres.

Profoundly complex mechanomolecular pathways transmit myofascial tension over the entire muscle fiber surface, from generation in actomyosin filaments of sarcomeres to the enveloping connective tissues $^{(4-9)}$. Thus, the properties of muscle fibers and their fascial networks are both integrally vital contributors to passive myofascial mechanics ${ }^{(7)}$. During increasing imposed stretching (or compression), as seen in the passive stress-strain curve of muscle fibers, the initial ("toe") static ("linear phase") response transitions into the succeeding dynamic ("non-linear phase") responses ${ }^{(2,10)}$. The initial static 
phase of such material stiffness has elastic properties and may be considered "spring-like," because of the absence of hysteresis. After release of minimal imposed strain, an ideally-elastic spring returns its full force. However, on further strain, biological materials become viscoelastic and show hysteresis - that is, an incomplete return of force on release of $\operatorname{strain}^{(3)}$. Viscoelastic responses also reflect the velocity of imposed deformations - that is, specific time- and rate-dependent behaviors (Table 1).

The lumbar multifidus is reviewed as a primary stabilizing muscle in the spine, rather than one having a mainly active movement function. By virtue of its specialized passive stiffness properties, the multifidus is an important example of HRMT functionality in the lumbar spine. The HRMT property may contribute to variability in normal function such as innate athletic performance or predisposition to particular biomechanically-induced clinical syndromes of pain and low-back disorders. In this regard, only ankylosing spondylitis (AS) is exemplified. A predisposition to this often progressive deforming disorder of vertebrae and sacroiliac joints is suspected in the presence of innate axial myofascial hypertonicity and its biomechanical consequences of excessive force concentrations and transmissions ${ }^{(11,12)}$.

\section{BIOMECHANICAL CONCEPTS}

\section{Mechanobiology and Tissue Material Properties Relevant to Passive Myofascial Tone}

Biological materials have biomechanical properties that depend on the chemical composition and structural organization of those materials. Resting [central nervous system (CNS)-independent] muscle, in addition to its specialized ability to actively contract and generate great tensile forces, displays such passive material properties ${ }^{(13)}$. All cells have cytoskeletal networks of microfilaments, including rigid actin, and non-muscle myosin motors that generate internally-integrated tension ("tensegrity") that determines mechanical behaviors under various loading scenarios ${ }^{(14-16)}$. In addition, external forces are propagated from the cell-surface integrin molecules to the nucleus along pre-stress pathways, as dictated by the degrees of tension in the cytoskeletal elements ${ }^{(15)}$. The architectural and compositional organization of materials (for example, size, shape, and density) confer additional specialized properties at various molecular, cellular, tissue, and organ levels ${ }^{(16)}$.

Biological materials can undergo further adaptations or optimization of responses by complex interactions with environmental signals, depending on genetic factors. Furthermore, body tissues undergo growth and remodeling in response to biomechanical forces. Notably, bone changes in shape, density, and stiffness in response to mechanical loads ${ }^{(17,18)}$.
Vascular cells also adapt to varying shear stresses and pressures ${ }^{(19,20)}$. Thus, tissues respond to mechanical signals that are translated at a molecular level into cascades of biochemical events ${ }^{(14-16)}$.

Accordingly, abnormal mechanical loading can alter cellular function and change the composition and arrangement of the extracellular matrix. Chronically unfavorable loading conditions eventually lead to tissue or organ pathologies - for example, enthesopathy, tendinopathy, osteoarthritis, and even atherosclerosis $^{(21-25)}$. Hence, it becomes imperative to understand the complex mechanobiology of how cells sense external forces and how tissues adapt or maladapt to forces.

Cells and tissues also engage in coupling mechanisms over various size scales, such as the intimate covering of muscle fibers and bundles by continuous networks of collagenous fibrils and other extracellular matrix molecules ${ }^{(5-9)}$. In myofascia, such coupling influences the intrinsic material properties of each tissue component; however, this area of mechanobiology is still largely undefined ${ }^{(7,16)}$. Passive stiffness properties of lumbar multifidus fibers and fiber bundles are reviewed in this paper. However, scant research has been done on intrinsic tensional properties of human longitudinal ligaments ${ }^{(26)}$ or their functional role in stabilizing the spine.

\section{Molecular Factors Contributing to Passive Resting Myofascial Tone and Tensegrity}

Passive resting [electromyography (EMG)-silent] myofascial tone is a static tension derived from its CNS-independent material properties, as previously reviewed ${ }^{(1-3,27,28)}$. Conversely, CNS-stimulated muscle tone results from active contractions ${ }^{(3,13,27,28)}$, which generate far greater tension and are associated with clearly evident motor unit potentials on $\mathrm{EMG}^{(1-3)}$. The active muscle tension is needed to exert movements and to generate increased static resistance. The total force developed within a contracting muscle is the sum of its passive (low-level) and active (predominant) myofascial components ${ }^{(29)}$.

The 1968 pioneering experimental study by Hill(30) demonstrated a short-range (that is, static) elastic component (SREC) in the isolated denervated ("resting") frog sartorius muscle. After application of slow, small stretches $(0.2 \%$ of isometric length), the tensile force was estimated to be about $150 \mathrm{~g}$ per $100 \mathrm{~g}$ of this long, columnar-shaped muscle. Hill ${ }^{(30)}$ proposed the cross-bridge theory of SREC: that it results from molecular elasticity of the attachments of low-level cycling of thick myosin heads binding to thin actin filaments in sarcomeres of muscle fibers. Further research on resting muscle tension supports its static elastic properties and is consistent with, although not proof of, the molecular cross-bridge theory ${ }^{(31-33)}$.

Actin and myosin filaments are themselves intrinsically extensible. This mechanism was suggested 
to provide additional elasticity, especially when actively contracted ${ }^{(34-37)}$. However, that theory would not explain passive resting muscle tone at neutral lengths or at physiological extensions. Another molecular component, called titin, has been demonstrated to exert passive tension in cardiac muscle and in considerably stretched skeletal muscle $\mathrm{e}^{(38,39)}$. However, its elastic role in the multifidus muscle was not supported at neutral or extended lengths ${ }^{(40)}$. Similarly, the sarcolemmal connective tissue networks of collagen fibrils and other extracellular matrix molecules contribute to passive elasticity only after having been stretched beyond their physiological lengths ${ }^{(7)}$.

The tensegrity theory proposes that cytoskeletal filaments are interconnected structural elements within the cellular and tissue systems, which are linked together into a balance of forces that provides stability and permits efficient transfer of forces ${ }^{(14-16,41,42)}$. The balance of integrated tension (that is, tensegrity) is controlled and maintained by the process of mechanotransduction. This specialized receptor mechanism senses forces in cells and generates signals that are coupled with the imposed environmental signals, becoming integrated at molecular levels into biochemical responses ${ }^{(14-16)}$.

The tensegrity theory supports the HRMT model based on

- the generation of SREC within sarcomeres ${ }^{(30-33)}$.

- the hierarchical architectural organization of continuous or serially arranged myofibers in muscle ${ }^{(13,16)}$.

- the tension being transmitted along integrated fascial webs and networks within the cytoskeleton $^{(4-9)}$.

Endomysial tubes surround individual muscle fibers, and the coarser, stiffer perimysium separates bundles or fascicles of muscle fibers; by contrast, the epimysium surrounds the whole skeletal muscle ${ }^{(5-9-}$ ,43-45). The amount of connective tissue and the crisscross arrangement of the crimped collagen fibers further influence the transmission of passive resting myofascial tone during elongation ${ }^{(5,43-45)}$.

Although quantitative data on HRMT (tentative analog of the experimental SREC) are limited, a review of passive myofascial properties supports low-level tension and elastic reactive responses to minimal perturbations ${ }^{(1-3,28,30-33,46,47)}$. However, on passive movements greater than $0.2 \%-0.4 \%$ elongations beyond the resting length, the initial (static) elastic phase then transitions into a far lesser viscoelastic tension response ${ }^{(2,3,28,32)}$. The crossbridges are disengaged in that early movement phase within the physiological range, permitting a sliding ("friction") to occur between the actin and myosin filaments. The decrease in passive tension observed from the static to the early movement phase is analogous to phenomena also observed in physical friction and thixotropy. In both conditions, resistance ("inertia") and viscosity become less in the movement phase ${ }^{(3,32)}$.

However, upon further experimental elongation of fibers beyond their physiological range, passive tension again increases significantly $(40,48)$. In that longstretch phase, the stiffer fascial filamentous responses prevail $^{(7)}$, far exceeding those earlier tensions of the elastic actomyosin bridging or the viscoelastic sliding phases respectively $(2,3,7,28,32)$

In the stress-strain curve, low-level elastic HRMT properties are reflected by the initial toe $(2,10)$, rather than by the stiffer late stretch segment $(2,3,40,48)$. The human analogy of late stretch stiffness may be the sensation of increased endrange resistance that an examiner feels when completing an assistive range-of-motion maneuver. At end-range, increased stiffness may be contributed by greater resistance from fascial tissues, muscle contracture, or reflex contraction ${ }^{(3)}$ rather than from passive sliding muscle fibers. Although the HRMT model applies mainly to the passive static component of muscle physiology, its chronic alterations may impose late effects by inducing myofascial remodeling; such a possibility would have to be determined in future study.

\section{Functional Relations to Resting Myofascial Tone}

\section{Resting (Static) Myofascial Tone Mainly Helps to Stabilize Balanced Postures}

Conventional biomechanical models of stability deem that a synergy of forces is required, integrated by

- CNS stimulation of active muscle contraction,

- the passive osteoligamentous system, and

- the passive myofascial component ${ }^{(49-55)}$.

However, little or no active force is required to help maintain equilibrium in gravity-balanced positions ${ }^{(46,47,56)}$. Accordingly, assumption of balanced postures can economize the work of active contraction, reduce fatigue, and likely lessen various myofascial strain symptoms: that is, the advisability of minimal effort postures ${ }^{(56)}$.

Osteoligamentous instability of the lumbar spine has long been the major explanation for increased biomechanical risk of developing low back disorders ${ }^{(49-51)}$. By contrast, passive muscles and their biomechanical properties have not been investigated for their beneficial role in optimally stabilizing balanced postures. Notably, clinical and individual variations in muscle firmness or stiffness qualities can be confidently detected by manual and movement practitioners when examining patients in their static relaxed positions or in assisting movements that are initiated from the resting state. These maneuvers are reliable $\mathrm{e}^{(57,58)}$ and can assist in physical diagnosis 
and in a consideration of appropriate therapy for significant alterations of HRMT - for example, appropriate mobilizing, selective strengthening, or other prescribed exercises - which deserve greater future attention.

As with other physiological traits, axial HRMT is suspected to have a genetic ("adaptive") individual variability (polymorphism) in the population. Considering biological variations, a small subset of people would be expected to have an intrinsic insufficiency of such a trait, and a similarly small subset would have an excess tonicity ${ }^{(59)}$. An insufficiency of axial myofascial tonicity was proposed to increase predisposition to adolescent idiopathic scoliosis, and substantial hypertonicity has been hypothesized to lead to $\mathrm{AS}^{(59)}$, as will be described shortly.

\section{Passive Myofascial Tone Has Not Been Incorporated into Models of Lumbar Stability}

Structural instability is currently recognized as an explanation for lumbar spine dysfunctions and pain ${ }^{(4-51)}$. However, excessive passive stiffness or needless co-activations also impose load penalties and adverse consequences on joints and bony attachment sites (entheses) and have not been sufficiently addressed. A demanding technical precision is needed to quantify low-level resting tension and to differentiate individuals having variations in such tone, a situation that has not often been studied ${ }^{(1,2,46,47)}$. Although the biomechanical model of HRMT has not yet been wellquantified, it is proposed to be an additional passive component ${ }^{(1)}$ within the conventionally-accepted spinal stabilizing systems ${ }^{(49-51,53)}$.

Various CNS-activated programs of motor control fitness and enhanced coordination of movements are therapies often prescribed to achieve desirable spinal stiffness and stability. However, the assessment and management of altered passive myofascial tonicity has not been well addressed ${ }^{(3)}$. Importantly, most modern occupational and recreational activities involve static or near-static minimal-effort postures that could be optimally stabilized by proper positioning and passive tone ${ }^{(1,56)}$.

After removal of muscle, the osteoligamentous lumbar spine was estimated to support a $90 \mathrm{~N}(9.0 \mathrm{~kg})$ compressional load ${ }^{(60)}$, which certainly is not sufficient to support the trunk. Thus, paralumbar muscles - and particularly the multifidus - are required to provide essential global and local support ${ }^{(61)}$. Notably, resting (EMG-silent) lumbar muscles are usually sufficiently stiff to stabilize the spine in relaxed, gravity-balanced sitting or standing postures ${ }^{(1-3)}$. As mentioned earlier, the tension of passive static tone is low ${ }^{(28,46,47)}$ and even less in movement ${ }^{(32)}$. The tension of resting limb muscles is estimated to be less than $1 \%$ of maximal voluntary contractions ${ }^{(30-33)}$, but may be $3 \%-5 \%$ for the lumbar multifidus ${ }^{(13,40,48, a)}$.

a Lieber RL, PhD. July 27, 2010. Personal communication.

\section{Tissue Connectivity and Relation to HRMT}

Connected tissues, such as "muscle-tendon/ligament-bone" units, have passive material properties that reflect their respective components and influence efficient force transmission and biomechanical integrity ${ }^{(5-9,25,62)}$. Furthermore, tissue interfaces of such units are optimally designed to minimize stress concentrations $^{(5,8,25)}$. Chronic overloading of these integrated units can increase the risk of acquiring enthesopathy syndromes and low back disorders, as indicated for AS. Congenital abnormalities can contribute to disorders of tissue connectivity, such as the marked ligamentous (hyper)laxity found in Marfan syndrome, caused by fibrillin-1 dysfunctions ${ }^{(63)}$. By comparison, increased passive stiffness of neck muscles is often noted in people having tension-type headache $(\mathrm{TTH})$ and torticollis ${ }^{(1,3,54)}$.

The interface transitions are structurally designed both to dampen and to efficiently transmit forces without undue damage to the involved tissues ${ }^{(7,25)}$. The interface structures incorporate gradients of material stiffness (that is, graded elastic moduli) of the specialized tissues. Tissue connections having the proper transition gradients in their mechanical properties resist structural damage more than a homogeneous tissue unit does ${ }^{(62)}$. When a normally-toned muscle is attached to a ligament or bone, it can theoretically be considered an extensible-stiff gradient of the connectivity unit. Normally-relaxed muscle is relatively soft and extensible; it can efficiently dissipate stress concentrations by transferring or absorbing them. However, if the static resting muscle were to be clearly hypertonic, the normal extensible-stiff connectivity would become more of a stiff-stiff transition. Notably, forces tend to follow the path of greatest stiffness within myofascial tissue $^{(9)}$. Thus, stiffer muscles less effectively absorb or distribute forces, thereby transmitting greater stresses to tendons or ligaments and to bony enthesis sites, which serve an anchoring role ${ }^{(25)}$. Examples in athletics of adverse consequences from stiffer muscles are the more frequent regional injuries incurred after fatigue $^{(64)}$ or when insufficient warm-up stretching is done by someone with hamstring tightness ${ }^{(65)}$.

Additionally, all tissues have a mechanical tolerance to stress or an intrinsic strength. If the forces acting upon tissues chronically exceed the tolerance limits of those tissues, injury reactions are more likely to ensue $^{(25,66)}$. Chronically enhanced stress concentrations predispose to greater tissue injury and lead to maladaptive repair processes, including fibrous dysplasia, calcifications, and new bone formation at characteristic enthesis sites ${ }^{(25)}$, found prominently in $\mathrm{AS}^{(11)}$.

\section{The Lumbar Multifidus Is the Core Muscle Stabilizer in the Spine}

The intact human lumbar spine provides the essential stability (stiffness) needed to maintain the 
unique upright posture of humans - at rest and in dynamic functions ${ }^{(61,67-70)}$. As the structural bridge between the relatively rigid thoracic spine and the large fused sacral platform, it transfers considerable forces. The lumbar multifidus is the largest and strongest of the low back paravertebral muscles that cross the lumbosacral level, but its exact morphology and role are complex and not fully determined ${ }^{(71-75)}$. Nevertheless, rather than its being a prime mover, the specialized multifidus, and especially its deep fibers, are largely credited with postural stabilization of the lumbar spine ${ }^{(40,61,72-79)}$.

The lumbar spine is stabilized and moved by sets of muscles that have varying biomechanical roles ${ }^{(67-70)}$. Circumferentially, the paralumbar muscles are arranged from those closest to the spine ("local" or "core") to those located peripherally in the pelvic and abdominal perimeters ("global" or "shell"). Optimal function requires a balanced coordination of all core and peripheral muscle groups. The core muscles provide mainly segmental stability; the more peripheral muscles control mainly global movements and general stabilization of the trunk ${ }^{(61,79)}$. An insufficiency of one group, such as the core multifidus, places excessive demand on the peripheral muscles to provide chronic stabilization.

Besides biomechanical functions, the core and peripheral muscle groups show differences in histology, neurophysiology, and tolerance to sustaining low-level tensioning. Posterior muscles that are more superficial, such as the iliocostalis, are more adapted to controlling mobility and large perturbations in posture. They are mainly phasic, type 2 ("fast-twitch") muscles designed for rapid motion rather than for chronic postural stabilization ${ }^{(79)}$. Their architecture and orientation are also biomechanically leveraged for global movements and dynamic positioning ${ }^{(67-70)}$.

The question of whether maximum contractile tension differs by muscle fiber type is controversial and difficult to determine ${ }^{(13)}$. To properly compare muscles of different sizes (or their fibers and bundles), the force needs to be normalized to the physiological cross-sectional area (PCSA) - that is, the volume divided by the length. That value is called the "specific tension": the force of contraction per unit area of fiber, bundle, or muscle, which is expressed as newtons per square centimeter or as kilopascals in SI units $(1 \mathrm{~N} /$ $\mathrm{cm}^{2}=10 \mathrm{kPa}$, in SI conversion). A pascal is a force of 1 newton per square meter.

The concept of HRMT actually refers to the specific tension (tone) of the muscle, normalized for its size. Lieber ${ }^{(13)}$ considers that the best information currently available on this issue derives from a study that showed that fast fibers generate just slightly more tension than slow fibers $\mathrm{do}^{(80)}$. As recently reviewed ${ }^{(79)}$, values for specific tension vary over a large range, between $22.5 \mathrm{~N} / \mathrm{cm}^{2}$ and $100 \mathrm{~N} / \mathrm{cm}^{2}$. Unfortunately, comparison data for the specific tension of postural HRMT are not available. Accurate measurements promise rewards for clinical practice and research.

\section{Lumbar Multifidus Type Specificity and Proprioceptive Function}

The multifidus is the most medial (core) muscle

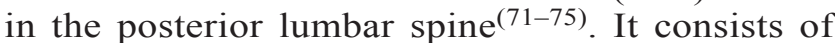
repeated short fascicles with attachments spanning the L1-L2 to L5-S1 intervertebral levels. The lumbar multifidi are mainly type 1 (slow-twitch) tonic postural muscles ${ }^{(81)}$, particularly the deep fibers ${ }^{(82)}$. The lumbar type 1 fibers have significantly greater diameter than the type 2 (fast-twitch) fibers do, suggesting that the type 1 fibers have a particular functional role ${ }^{(81-84)}$

The concentration of spindles in small, short muscles acting across a joint in parallel with vastly larger and longer muscles may serve an important sensory feedback role and may function as "kinesiologic monitors"(85-87). The spindle concentrations in the small muscles of such parallel muscle combinations were estimated to be greater than those in the larger and longer muscles by a factor of between 5 and $7^{(86)}$. The deepest fibers of the multifidus may serve such a proprioceptive function ${ }^{(87)}$. Deep, short fibers such as these may also act as biomechanical stabilizers of movement at the joint surface and may behave as "dynamic ligaments"(76). The multifidus has a greater density of muscle spindles in the fascicles closer to the facet joints ${ }^{(86)}$. Accordingly, compared with other back muscles, the lumbar multifidus is suited to provide passive and active stability to the lumbar spine ${ }^{(88)}$.

The multifidus is also considered to display arthrogenic muscle inhibition, which is attributed to damage in associated joint structures ${ }^{(89,90)}$. Such reflex motoneuron inhibition was generalized to explain muscle weakness and atrophy from all forms of joint damage ${ }^{(89)}$. Sensory facilitation may also contribute to increased muscle stiffness, at least as inferred from muscles around the ankle joint ${ }^{(90)}$.

\section{Fascicular Orientation of the Lumbar Multifidus}

Each ipsilateral lumbar multifidus is divided by cleavage planes into 5 bands ("myotomes"), which are unisegmentally innervated and arise from the respective lumbar levels (Fig. 1) ${ }^{(71-74)}$. The individual bands consist of a series of fascicles, layered in depth. Collectively, the deeper fascicles attach 2 intervertebral levels below their origins, except for the deep L5 lamina fascicles, which bridge only the L5-S1 intervertebral level [Fig. 1(B)]. The deepest, most medial, and shortest fascicles arise from the lamina of each L1 - L5 at their caudal dorsal surfaces [Fig. 1(B)]. The L1 - L4 laminar fascicles insert onto lower mammillary processes, 2 levels below. However, the L5 lamina fascicles insert above the 1 st dorsal sacral foramen [Fig. 1(B)], because no 

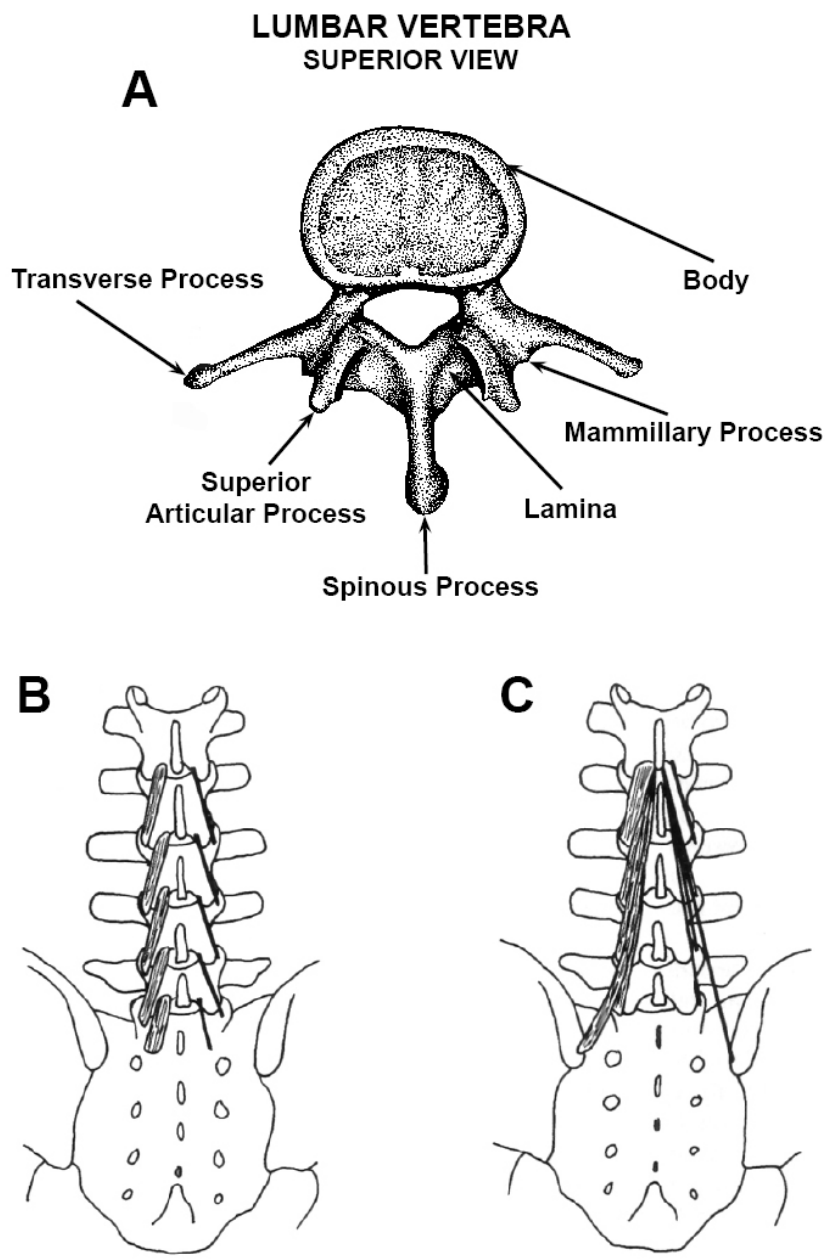

FIGURE 1. Lumbar vertebra and multifidus fascicles. (A) Superior view of the lumbar vertebra shows posterior bony elements from which multifidus fascicles arise and insert, as also described in the text. (B) The deepest fibers of multifidus bands arise from the caudal dorsal aspect of the L1 - L5 laminae and insert on lower mammillary processes. The L1 - L4 laminar fascicles span two intervertebral discs, but the L5 fascicles span only the L5 - S1 level, as shown. (C) Multifidus fascicles are shown only for those arising from L1. The more superficial multifidus fibers that arise from the spinous processes insert $3-5$ levels below their muscle origins on L1-L5, and have longer lengths than the fibers that arise from the laminae. The longest and most superficial bands of fascicles stem from the spinous processes of L1-L3. Reproduced with the permission of Elsevier Churchill Livingstone, from Clinical Anatomy of the Lumbar Spine and Sacrum (Fig. 9.4, panels A and B, p. 102) ${ }^{(73)}$.

mammillary process exists below that of $\mathrm{S} 1$ to insert upon. Such short segmental bridging provides stiffness and translational stability to the lumbar spine.

More superficial fascicles arise along the caudal edges of the spinous processes, extending from their base and lateral surfaces [Fig. 1(C)] and attach up to 5 intervertebral levels below their origins. The yet most superficial fascicles originate from a common tendon along the caudodorsal tip of the spinous processes, which are also the most laterally oriented fascicles. They cross the sacroiliac joints, having attachments on the posterior superior iliac spine ${ }^{(70)}$. The multifidus myofibers are arranged in a multi-pennate fashion, which allows for greater strength and stiffness ${ }^{(40,62,74)}$.

Some of the deepest multifidus fibers originate from the dorsal capsules of the zygapophysial ("facet") joints ${ }^{(67,73)}$. Anteriorly, the fibrous capsules of the facet joints are replaced by the ligamentum flavum $^{(73,75)}$. Such encapsulating elastic attachments on the zygapophysial joints may protect inner capsular tissues from being nipped between the cartilage surfaces during extension ${ }^{(70,73,75)}$.

\section{Multifidus Architecture and Passive Stiffness Enhances Stabilization of the Lumbar Spine}

In lateral view, the lumbar multifidus is oriented mainly caudally and vertically; the deeper fascicles have slightly anterior angulation ${ }^{(67-69,73,75)}$. Such axial orientation enhances stability against axial translation in the lumbar spine. In extension, the muscles are shortened and less stiff ${ }^{(40,48)}$, but the facet joints become more compressed and stable. In the fully extended position, the facet joints are in a "closed pack" position and lose mobility. In flexion, the deeper multifidus is lengthened and becomes stiffer, helping to regain control of anterior vertebral translation ${ }^{(48,67)}$.

The large physiological cross-sectional area of the lumbar multifidus and its low fiber-length-tomuscle-length ratio is designed to function as a passive stabilizer of the spine ${ }^{(40,48)}$. Also, the actomyosin filaments interdigitate completely throughout the sarcomere lengths corresponding to the physiological range of motion ${ }^{(48,91)}$. Furthermore, the overlapping of myofilaments is restricted only to the ascending portion of the length-tension curve ${ }^{(48)}$. Such unique filamentous interdigitation permits the multifidus to become passively stiffer as the spine assumes a forward-leaning posture ${ }^{(40,48)}$.

The stiffness of single fibers of the multifidus was estimated from its elastic modulus, which was calculated as the tangent slope of the stress-strain curve $^{(40)}$ (Table 1). This value was determined for fibers stretched from sarcomere lengths of $2.0 \mu \mathrm{m}$ (as in prone position) to $4.25 \mu \mathrm{m}$ (mechanical failure) $(40,91)$. In such imposed lengthening, the elastic modulus for single multifidus fibers was similar to that for single fibers of the longissimus and iliocostalis erector spinae muscles ${ }^{(40)}$. However, the elastic modulus of multifidus fiber bundles was nearly 50\% greater than for bundles from the two erector spinae muscles ${ }^{(40)}$. The molecular mass of titin did not differ between the single fibers of the various muscles, nor did the titin mass correlate with any of the elastic properties ${ }^{(40)}$. The difference in stiffness observed for the various fiber bundles was suggested to be attributable to muscle-specific extracellular matrix properties. 
In full flexion, the lumbar extensors are normally silent when the spine is in a ligamentous support phase (known as the "flexion-relaxation phenomenon") ${ }^{(92)}$. Also, when standing in a gentle extended lordotic posture, the lumbar extensors have minimal to no functional activity ${ }^{(1)}$. Support is then borne by the anterior abdominal muscles tensing eccentrically and by passive structures such as the iliofemoral ligament. Reciprocal inhibition of the multifidus from eccentric abdominal muscular contraction may also occur in this position. By its eccentric contractions, the multifidus also adds stability and smoothness to the controlled speed of movements in forward flexion of the spine. However, the role of the lumbar multifidus in movement or active contraction functions is not reviewed.

\section{Person-Related Influences on HRMT and Relevance to AS}

\section{Clinical, Demographic, and Physical Activity Relations to Resting Myofascial Tone}

Certain factors - such as age, exercise, and environmental cues (posture and psychological tension)-may influence myofascial tone ${ }^{(55,93-95)}$. Increased resting muscle tone has been noted in TTH and torticollis ${ }^{(1,3)}$, tight thigh or calf muscles ${ }^{(3)}$, and $\mathrm{AS}^{(11,12,42,96)}$. Symptomatic and objective muscle stiffness is also associated with muscle contractures and other chronic muscular tightness syndromes often encountered in rehabilitation settings $(3,52,54)$.

Aging may influence myofascial tone by reducing the number of muscle fibers and their cross-sectional area ${ }^{(97)}$. The reduced overall muscle mass (atrophy) in senility ${ }^{(97,98)}$ may decrease extensibility on passive stretch $^{(99)}$. The connective tissue content of muscle increases with age ${ }^{(99)}$ as lost muscle tissue is replaced by fibrous connective tissue, thereby increasing passive muscle stiffness. An aging effect on increased stiffness was demonstrated during imposed rotation of the ankle to determine calf muscle properties ${ }^{(100)}$. However, such studies do not focus on the static elastic component of resting muscle tone, as measured in relaxed quiet standing ${ }^{(46,47)}$. Further studies of aging effects on HRMT are needed.

Immobilization and decreased use also lead to reduced muscle volume, structure, and function ${ }^{(101,102)}$. By contrast, increased use can stimulate greater muscle strength and type 2 fiber size ${ }^{(103)}$. In a study of effects of 3 months of maximal or submaximal physical rehabilitation in patients with chronic low back pain, trunk extension strength increased about $20 \%$ and type 2 multifidus fiber size increased about $10 \%$ in men (both $p<0.05$ ). Women had an increase in extension strength of about $10 \%(p=0.16)$, but not an impressive increase in type 2 multifidus fiber size ${ }^{(103)}$. Limited muscle-biopsy studies have been performed in relation to comparative exercise types such as resistance and eccentric ergometer training. Both exercise types are beneficial for the elderly with regard to muscle functional and structural improvements ${ }^{(104)}$.

As with many other tissues within the body, muscle displays significant plasticity in its geometry ${ }^{(105)}$. Heavy resistance training in young adults is believed to increase the fascicle angle; high-speed training, without weight training, has been associated with increased fascicle length and a reduction in angles, at least in the vastus lateralis ${ }^{(105)}$. No data were found with respect to effects on HRMT of such varied exercise conditions. Nevertheless, patients with symptomatically or objectively stiff, sore, or tight muscles should continue to be advised to perform clinically appropriate stretching and other exercises.

After acute or subacute lumbar injury, decreased lumbar multifidus girth was found ipsilateral to the pain at the clinically determined level of symptoms ${ }^{(106)}$. If the lumbar multifidus and its stabilizing tonic role in the spine are not restored therapeutically with specific retraining, recovery to fully normal size may not occur naturally on remission of painful symptoms ${ }^{(78,107-110)}$. Deficient lumbar multifidus function predisposes to increased risk and recurrence of low back pain, more work days lost, and increased use of medical services to treat recurrences ${ }^{(108)}$. The literature on the role of the lumbar multifidus in spinal stability and its relation to low back pain was critically evaluated ${ }^{(110)}$.

\section{Biomechanical Factors in Enthesopathy and Relevance to AS}

Non-inflammatory abnormalities of bony attachment sites (entheses) are called enthesopathies. Most often, they result from localized forces chronically exceeding the intrinsic biomechanical properties (tolerances) of the tissue units, as seen commonly in lateral epicondylitis ("tennis elbow") $)^{(25)}$. Although the body may naturally repair damage from repeated micro-injury, the healing may be maladaptive-for example, fibrosis, dysplasia, or calcification ${ }^{(25)}$. Inflammatory changes may also be induced at entheses, which is then called "enthesitis"(25) and which may require more aggressive localized therapy.

The chronic and often progressive spinal deformity condition that is AS was proposed to be contributed by a constitutional trait of axial myofascial hypertonicity $(11,12,42,59,96)$. The AS patient usually presents with symptomatic and objective stiffness and tight low back muscles, which typically improve modestly with stretching maneuvers and warm baths or showers ${ }^{(11)}$. Pathologically, the hallmark feature is a characteristic localization of vertebral enthesopathy lesional sites ${ }^{(11,12)}$. Whether the lesional process in AS results primarily from biomechanicallyinduced enthesopathy mechanisms, or whether it may require an additional inflammatory contribution (enthesitis), is not known ${ }^{(11,12)}$. Immunological mechanisms are generally considered to cause inflammation at the characteristic enthesis sites in 
$\mathrm{AS}^{(12)}$. However, we propose an alternative scenario in which excess stress concentrations and transmissions are the initial and primary contributing factors to the development of the enthesis lesions, which precede the onset of inflammatory reactions ${ }^{(11,12)}$. In theory, the chronic biomechanical stresses could predispose to increased micro-injury, leading to the consequent maladaptive tissue repair reactions and even to activation of innate immunological pathways that might cause inflammation ${ }^{(12,42)}$.

In the early development of AS, however, a synergy may occur between altered biomechanical and immunological activation pathways that may conjointly be needed to bring about localization of lesions at the characteristic enthesopathy sites. The sequence of the biomechanical vis-à-vis the inflammatory pathways needs to be determined in this disease process. Chronically increased stress concentrations could stimulate mechanoreceptors to cause tissue maladaptations, as observed in the structural lesions of the vertebral column in AS-for example, abnormal calcifications, new bone formation, and fusion across spinal levels ${ }^{(11)}$. It is not known, however, if inflammation may be a biologically intrinsic reaction within such biomechanically-induced maladaptive tissue responses or a separate component ${ }^{(42)}$. Alternatively, inflammation may be a separate adaptive response to chronically increased stress settings. Perhaps pain serves to reduce physical activity with the goal of protecting the spinal cord against greater injury, given that this structure is crucial to ultimate survival. Further research on the sequence of causal pathways in AS is needed, including study of the relationships of HRMT with tissue connectivity and the chronic effects of excessive stress concentrations on the localization of enthesopathy lesions.

Although axial myofascial hypertonicity was proposed to increase susceptibility to $\mathrm{AS}^{(11,12)}$, the possibility of muscle hypertrophy was neither addressed nor suggested. In fact, in later stages of AS, atrophy of paralumbar and psoas muscles occurs at a stage when the intervertebral ligaments are calcified and spinal mobility is markedly decreased ${ }^{(111)}$. The atrophy process likely results from disuse, given that neither impingement nerve root nor intervertebral disc disease appears to be increased in AS. Also, the axial myofascial hypertonicity in AS is not currently postulated to affect particular local, as compared with global, stabilizers of the lumbopelvis ${ }^{(96)}$. Such specific biomechanical data are not yet available to interpret. Although the predisposition to AS is clearly inherited $^{(11,12)}$, its pathogenesis is not yet known. If indeed a myofascial predisposition exists in AS, one possible mechanism could be an excessive intrinsic generation of tension-that is, a polymorphism of sacromere-derived HRMT.

Alternatively, might chronic extrinsic overloading of fascial tissues, because of various biomechanical factors, be a main mechanism that alters viscoelastic properties ${ }^{(112)}$ ? Could fascial stresses of those kinds generate pain by sensitizing densely localized nerve endings $(52,112)$, which could then possibly amplify HRMT? Once chronic low back aching, pain, and stiffness begin to manifest in the early course of AS (though not always as the first symptoms), then an interpretation of the underlying mechanism for hypertonicity (intrinsic versus pain-response) becomes difficult to decipher ${ }^{(11)}$. This question can, however, be determined scientifically in a controlled prospective study of asymptomatic adolescent and young adult siblings and children of AS patients. A followup study could determine whether axial myofascial hypertonicity might precede or follow the onset of pain symptoms and whether AS might likely be a result of axial myofascial hypertonicity.

\section{EPILOGUE}

\section{Intent of the Review: Dedicated Practitioners- Learners Can Become Thought Leaders}

The present review is intended to stimulate discussion, interest, and understanding of a complex physiological feature that is relevant to patient management, but that has not yet been reliably quantified. The curiosity of practitioners about HRMT and what they come to learn about it promise to help define its clinical relevance and to provide clues for research into its mechanisms. Theories about complex biological systems do not always derive from experimental research, but are often generated by the intuition of informed clinicians. In turn, such practical observations often stimulate basic investigations about the biological nature and progression of disease. Transdisciplinary approaches and examinations of problems from various perspectives or fields of investigation often yield novel hypotheses ${ }^{(113)}$. The National Institutes of Health are promoting the discipline of clinical and translational science as a melding of clinical studies with basic science research for a more productive investigative approach (visit http://nihroadmap.nih.gov/clinicalresearch/overviewtranslational.asp for an overview).

The understanding of complex processes advances in stages. Knowledge may be described simply as knowing events, facts, or principles related to states or systems. But knowledge incorporates an orderly synthesis and is broader in scope than a random database of information alone. Wisdom may be considered to be the next stage of understanding. It is the synthesis of greater knowledge or learning with sound experienced judgments to interpret what is likely to be true or valid. Intuition is yet a higher cognitive level, being the ability to gain insight about complex processes, without direct deduction from already-established premises or induction from proven examples. Intuition is an inferential and synthetic thought system, 
guided by informed expectations about perceived factors in a complex process. Albert Einstein said, "The only real valuable thing is intuition."

Concepts of HRMT are supported by sound biomechanical principles, but are yet more intuitive than quantitatively established. Measuring the core components of complex processes, such as those in HRMT, can prove to be technically difficult or unattainable. Nevertheless, clinical observations on the altered status or dysfunctions of HRMT can provide clues to its mechanisms. As in physics, "thought experiments" can generate new concepts or models (qualitative descriptions) that may then stimulate progress in quantitative or experimental research.

\section{Scientific Understanding of Natural Processes}

An understanding of natural processes results from a universe of observations and experimental data (Fig. 2). Early observational understandings lead to theories or models of processes, which then generate initial predictions and guide experimentation ${ }^{(114)}$. Quantitative simulations of theoretical models also contribute to more refined understandings of natural processes ${ }^{(79)}$.

\section{Principles of Systems Biology and Physiology}

The rationale of this review may be analogous to that of systems biology and physiology. Those approaches aim to achieve an integrative, multidisciplinary synthesis of complex processes, a goal that also applies to current challenges in deriving valid concepts of HRMT or resting myofascial tone (RMT). In systems research, fundamental principles are applied to derive interdisciplinary models or investigative approaches to the complex processes. The objective is to create an integrated and simplified model of understanding of the processes. Concepts of

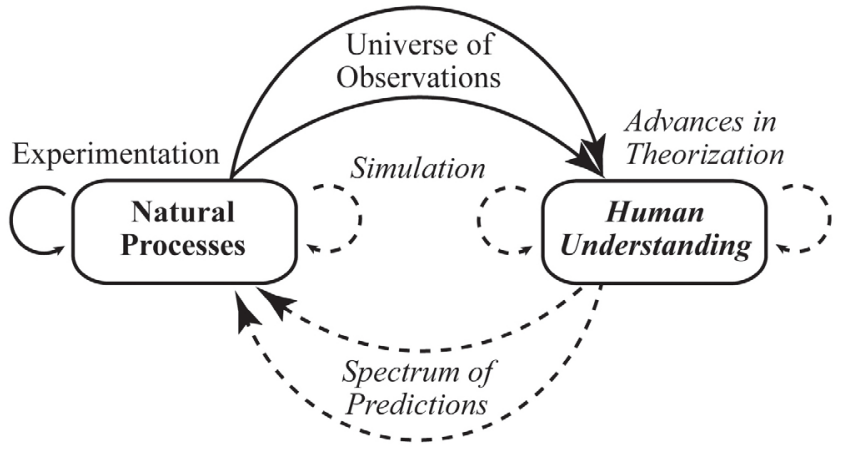

FIGURE 2. The relations of scientific understanding to natural processes. Scientific understanding of natural processes involves a complex sequential synthesis and refinement of theories, based on universes of observations, experimentation, and simulation, in addition to validity of predictions. Based on The Computational Beauty of Nature ${ }^{(114)}$. universal design are incorporated into the engineering components of systems research, to the extent that those mechanical principles are biologically applicable. In the present review, the integrated tension ("biotensegrity") of cells, tissues, and regions of the body ${ }^{(14-16,41)}$ is presented as an essential property in biomechanics and as a fundamental tenet in the stabilizing role of RMT.

The quantitatively subtle entity of RMT is essentially overlooked in human biomechanics ${ }^{(49-51)}$ and in experimental research on muscle physiology ${ }^{(13,32)}$. However, this entity is persuasively supported in the literature review. In clinical and manual practice, the physical features of HRMT are recognized. However, relevance of HRMT is not currently being well addressed in certain musculoskeletal disorders and deserves greater future attention. Variations of anthropomorphic and biological design features among individuals within populations (polymorphisms) are generally believed to have a basis in human evolution and to have been influenced by environmental adaptations. Assuming such, the HRMT trait and its biomechanical role are likely to be intrinsically integrated within basic systems in the body. Its future study presents considerable challenges for clinical understanding and research, but promises commensurate rewards.

\section{ACKNOWLEDGMENTS}

We thank Becky Hawkins for her invaluable technical assistance in completing this manuscript and Michael Berry, BS, for technical assistance with the work. Dr. Sam Betts and Professors Robert Schleip and Thomas Santoro provided expert critiques on the manuscript. Support for this project was provided by the Department of Medicine, University of Illinois College of Medicine at Peoria, and by the MTM Foundation.

\section{CONFLICT OF INTEREST NOTIFICATION}

The author declares that there are no conflicts of interest.

\section{COPYRIGHT}

Published under the CreativeCommons Attribution-NonCommercial-NoDerivs 3.0 License.

\section{REFERENCES}

1. Masi AT, Hannon JC. Human resting muscle tone (HRMT): narrative introduction and modern concepts. $J$ Bodyw Mov Ther. 2008;12(4):320-332. 
2. Masi AT, Hannon JC, Nair K. Response to letter to editor on human resting muscle tone (HRMT). J Bodyw Mov Ther. 2009;13(2):118-120.

3. Mense S, Masi AT. Increased muscle tone as a cause of muscle pain. In: Mense S, Gerwin RD, eds. Muscle Pain: Understanding the Mechanisms. Berlin, Germany: Springer-Verlag; 2010: 207-249.

4. Findley TW, Schleip R, eds. Fascia Research: Basic Science and Implications for Conventional and Complementary Health Care. Munich, Germany: Elsevier; 2007.

5. Purslow PP. Strain-induced reorientation of an intramuscular connective tissue network: implications for passive muscle elasticity. J Biomech. 1989;22(1):21-31.

6. Trotter JA, Purslow PP. Functional morphology of the endomysium in series fibered muscles. J Morphol. 1992;212(2):109-122.

7. Trotter JA. Functional morphology of force transmission in skeletal muscle: a brief review. Acta Anat (Basel). 1993;146(4):205-222.

8. Purslow PP. The structure and functional significance of variations in the connective tissue within muscle. Comp Biochem Physiol A Mol Integr Physiol. 2002;133(4):947-966.

9. Trotter JA. Structure-function considerations of muscle-tendon junctions. Comp Biochem Physiol A Mol Integr Physiol. 2002;133(4):1127-1133.

10. Levin SM. Human resting muscle tone (HRMT): narrative, introduction and modern concepts [J Bodywork Movement Ther. 12 (2008) 320-332] (comment). J Bodyw Mov Ther. 2009;13:117-118.

11. Masi AT, Benjamin M, Vleeming A. Anatomical, biomechanical, and clinical perspectives on sacroiliac joints: an integrative synthesis of biodynamic mechanisms related to ankylosing spondylitis. In: Vleeming A, Stoeckhart R, eds. Movement, Stability, and Lumbopelvic Pain: Integration of Research and Therapy. Edinburgh, Scotland: Churchill Livingstone; 2007: 205-227.

12. Masi AT, Savage LM. Integrated biomechanical influences on ankylosing spondylitis. In: Spondylitis Association of America (SAA). Spondylitis Plus. Sherman Oaks, CA: SAA; Summer 2009: 10-13. http://www.spondylitis.org/research/pdf/biomechanical ankylosing spondylitis.pdf. Accessed June 2010.

13. Lieber RL. Skeletal Muscle Structure, Function, and Plasticity: The Physiological Basis of Rehabilitation. 3rd ed. Baltimore, MD: Lippincott Williams and Wilkins; 2010.

14. Maniotis AJ, Chen CS, Ingber DE. Demonstration of mechanical connections between integrins, cytoskeletal filaments, and nucleoplasm that stabilize nuclear structure. Proc Natl Acad Sci U S A. 1997;94(3):849-854.

15. Hu S, Chen J, Butler JP, Wang N. Prestress mediates forces propagation into the nucleus. Biochem Biophys Res Commun. 2005;329(2):423-428.

16. Ingber DE. Tensegrity I. Cell structure and hierarchical systems biology. J Cell Sci. 2003;116(Pt 7):1157-1173.

17. Turner CH, Pavalko FM. Mechanotransduction and functional response of the skeleton to physical stress: the mechanisms and mechanics of bone adaptation. J Orthop Sci. 1998;3(6):346-355.

18. Mullender M, El Haj AJ, Yang Y, van Duin MA, Burger $\mathrm{EH}$, Klein-Nulend J. Mechanotransduction of bone cells in vitro: mechanobiology of bone tissue. Med Biol Eng Comput. 2004;42(1):14-21.

19. Owens GK. Role of mechanical strain in regulation of differentiation of vascular smooth muscle cells. Circ Res. 1996;79(5):1054-1055.

20. Williams B. Mechanical influences on vascular smooth muscle cell function. J Hypertens. 1998;16(12 Pt 2):1921-1929.

21. Chicurel ME, Chen CS, Ingber DE. Cellular control lies in the balance of forces. Curr Opin Cell Biol. 1998;10(2):232-239.

22. Grodzinsky AJ, Levenston ME, Jin M, Frank EH. Cartilage tissue remodeling in response to mechanical forces. Ann Rev Biomed Eng. 2000;2:691-713.

23. Ireland D, Harrall R, Curry V, Holloway G, Hackney R, Hazleman B, et al. Multiple changes in gene expression in chronic human Achilles tendinopathy. Matrix Biol. 2001;20(3):159-169.

24. Ross R. The pathogenesis of atherosclerosis - an update. $N$ Engl J Med. 1986;314(8):488-500.

25. Benjamin M, Toumi H, Ralphs JR, Bydder G, Best TM, Milz $\mathrm{S}$. Where tendons and ligaments meet bone: attachment sites ("entheses") in relation to exercise and/or mechanical load. $J$ Anat. 2006;208(4):471-490.

26. Tkaczuk H. Tensile properties of human lumbar longitudinal ligaments. Acta Orthop Scand. 1968;(Suppl 115):8-65.

27. Clemmesen S. Some studies on muscle tone. Proc R Soc Med. 1951;44(8):637-646.

28. Walsh EG. Muscles, Masses and Motion: The Physiology of Normality, Hypertonicity, Spasticity and Rigidity. London, UK: Mac Keith Press; 1992.

29. Gajdosik RL. Passive extensibility of skeletal muscle: review of the literature with clinical implications. Clin Biomech (Bristol, Avon). 2001;16(2):87-101.

30. Hill DK. Tension due to interaction between the sliding filaments in resting striated muscle. The effect of stimulation. $J$ Physiol. 1968;199(3):637-684.

31. Campbell KS, Lakie M. A cross-bridge mechanism can explain the thixotropic short-range elastic component of relaxed frog skeletal muscle. J Physiol. 1998;510(Pt 3):941-962.

32. Lännergren J. The effect of low-level activation on the mechanical properties of isolated frog muscle fibers. J Gen Physiol. 1971;58(2):145-162.

33. Woledge RC. Filamentary resting tension and latency relaxation. In: Sugi H, ed. Molecular and Cellular Aspects of Muscle Contraction. New York, NY: Kluwer Academic/Plenum Publishers; 2003.

34. Huxley HE, Stewart A, Sosa H, Irving T. X-Ray diffraction measurements of the extensibility of actin and myosin filaments in contracting muscle. Biophys J. 1994;67(6):2411-2421.

35. Wakabayashi K, Sugimoto Y, Tanaka H, Ueno Y, Takezawa Y, Amimeya Y. X-Ray diffraction evidence for the extensibility of the actin and myosin filaments during muscle contraction. Biophys J. 1994;67(6):2422-2435.

36. Goldman YE, Huxley AF. Actin compliance: are you pulling my chain? Biophys J. 1994;67(6):2131-2133.

37. Takezawa Y, Sugimoto Y, Wakabayashi K. Extensibility of the actin and myosin filaments in various states of skeletal muscle as studied by X-ray diffraction. Adv Exp Med Biol. 1998;453:309-317.

38. Wang K, McCarter R, Wright J, Beverly J, Ramirez-Mitchel $\mathrm{R}$. Viscoelasticity of the sarcomere matrix of skeletal muscles. 
The titin-myosin composite filament is a dual-stage molecular spring. Biophys J. 1993;64(4):1161-1177.

39. Linke WA, Ivemeyer M, Olivieri N, Kolmerer B, Rüegg JC, Labeit S. Towards a molecular understanding of the elasticity of titin. J Mol Biol. 1996;261(1):62-71.

40. Ward SR, Tomiya A, Regev GJ, Thacker BE, Benzl RC, $\mathrm{Kim} \mathrm{CW}$, et al. Passive mechanical properties of the lumbar multifidus muscle support its role as a stabilizer. J Biomech. 2009;42(10):1384-1389.

41. Ingber DE. The architecture of life. Sci Am. 1998;278(1):48-57.

42. Masi AT, Walsh EG. Ankylosing spondylitis: integrated clinical and physiological perspectives. Clin Exp Rheumatol. 2003;21(1):1-8.

43. Borg TK, Caulfield JB. Morphology of connective tissue in skeletal muscle. Tissue Cell. 1980;12(1):197-207.

44. Rowe RWD. Collagen fibre arrangement in intramuscular connective tissue. Changes associated with muscle shortening and their possible relevance to raw meat toughness measurements. Int J Food Sci Tech. 1974;9(4):501-508.

45. Rowe RW. Morphology of perimysial and endomysial connective tissue in skeletal muscle. Tissue Cell. 1981;13(4):681-690.

46. Loram ID, Maganaris CN, Lakie M. The passive, human calf muscles in relation to standing: the non-linear decrease from short range to long range stiffness. $J$ Physiol. 2007;584(Pt 2):661-675.

47. Loram ID, Maganaris CN, Lakie M. The passive, human calf muscles in relation to standing: the short range stiffness lies in the contractile component. J Physiol. 2007;584(Pt 2):677-692.

48. Ward SR, Kim CW, Eng CM, Gottschalk LJ 4th, Tomiya A, Garfin SR, et al. Architectural analysis and intraoperative measurements demonstrate the unique design of the multifidus muscle for lumbar spine stability. J Bone Joint Surg Am. 2009;91(1):176-185.

49. Panjabi MM. The stabilizing system of the spine. Part I. Function, dysfunction, adaptation, and enhancement. $J$ Spinal Disord. 1992;5(4):383-389,397.

50. Panjabi MM. The stabilizing system of the spine. Part II. Neutral zone and instability hypothesis. J Spinal Disord. 1992;5(4):390-397.

51. White AA, Panjabi MM. Clinical Biomechanics of the Spine. 2nd ed. Philadelphia, PA: Lippincott; 1992.

52. Schleip RJ. Fascial plasticity - a new neurobiological explanation - part 2. J Bodyw Mov Ther. 2003;7(1):104-116.

53. Lardner R. Stretching and flexibility: its importance in rehabilitation. J Bodyw Mov Ther. 2001;5(4):254-263.

54. Schleip R, Naylor IL, Ursu D, Melzer W, Zorn A, Wilke HJ, et al. Passive muscle stiffness may be influenced by active contractility of intramuscular connective tissue. Med Hypotheses. 2006;66(1):66-71.

55. Onambele GL, Narici MV, Maganaris CN. Calf muscle-tendon properties and postural balance in old age. J Appl Physiol. 2006;100(6):2048-2056.

56. Hannon JC. Wartenberg. Part 3: Relaxation training, centration and skeletal opposition: a conceptual model. J Bodyw Mov Ther. 2006;10(3):179-196.

57. Nicholson L, Maher C, Adams R, Phan-Thien N. Stiffness properties of the human lumbar spine: a lumped parameter model. Clin Biomech (Bristol, Avon). 2001;16(4):285-292.
58. Chiradejnant A, Maher CG, Latimer J. Objective manual assessment of lumbar posteroanterior stiffness is now possible. J Manipulative Physiol Ther. 2003;26(1):34-39.

59. Masi AT, Dorsch JL, Cholewicki J. Are adolescent idiopathic scoliosis and ankylosing spondylitis counter-opposing conditions? A hypothesis on biomechanical contributions predisposing to these spinal disorders. Clin Exp Rhematol. 2003;21(5):573-580.

60. Lucas DB, Bresler B. Stability of the ligamentous spine. Technical report no. 40. Berkeley, CA: Orthopaedic Biomechanics Laboratory, University of California; 1961: 1-41.

61. Bergmark A. Stability of the lumbar spine. A study in mechanical engineering. Acta Orthop Scand Suppl. 1989;230:1-54.

62. Suresh S. Graded materials for resistance to contact deformation and damage. Science. 2001;292(5526):2447-2451.

63. Callewaert B, Malfait F, Loeys B, De Paepe A. Ehlers-Danlos syndromes and Marfan syndrome. Best Pract Res Clin Rheumatol. 2008;22(1):165-189.

64. Christina KA, White SC, Gilchrist LA. Effect of localized muscle fatigue on vertical ground reaction forces and ankle joint motion during running. Hum Mov Sci. 2001;20(3):257-276.

65. Woods K, Bishop P, Jones E. Warm-up and stretching in the prevention of muscular injury. Sports Med. 2007;37(12):1089-1099.

66. Maganaris CN, Narici MV, Almekinders LC, Maffulli N. Biomechanics and pathophysiology of overuse tendon injuries: ideas on insertional tendinopathy. Sports Med. 2004;34(14):1005-1017.

67. Macintosh J, Bogduk N. The biomechanics of the lumbar multifidus. Clin Biomech. 1986;1(4):205-213.

68. Bogduk N, Macintosh JE, Pearcy MJ. A universal model of the lumbar back muscles in the upright position. Spine (Phila Pa 1976). 1992;17(8):897-913.

69. Macintosh JE, Pearcy MJ, Bogduk N. The axial torque of the lumbar back muscles: torsion strength of the back muscles. Aust N Z J Surg. 1993;63(3):205-212.

70. Kay A. An extensive literature review of the lumbar multifidus: biomechanics. J Man Manip Ther. 2001;9(1):17-39.

71. Macintosh J, Valencia F, Bogduk N, Munro R. The morphology of the human lumbar multifidus. Clin Biomech. 1986;1(4):196-204.

72. Kay A. An extensive literature review of the lumbar multifidus: anatomy. J Man Manip Ther. 2000;8(3):102-114.

73. Bogduk N, Endres SM. Clinical Anatomy of the Lumbar Spine and Sacrum. 4th ed. New York, NY: Elsevier/Churchill Livingstone; 2005.

74. Danneels L. Clinical anatomy of the lumbar multifidus. In: Vleeming A, Mooney V, Stoeckhart R, eds. Movement, Stability and Lumbopelvic Pain: Integration of Research and Therapy. Edinburgh, Scotland: Churchill Livingstone; 2007: 85-94.

75. Lonnemann ME, Paris SV, Gorniak GC. A morphological comparison of the human lumbar multifidus by chemical dissection. J Man Manip Ther. 2008;16(4):E84-E92.

76. Donisch EW, Basmajian JV. Electromyography of deep back muscles in man. Am J Anat. 1972;133(1):25-36.

77. Ng JK, Richardson CA, Jull GA. Electromyographic amplitude and frequency changes in the iliocostalis lumborum and multifidus muscles during a trunk holding test. Phys Ther. 1997;77(9):954-961.

78. Freeman MD, Woodham MA, Woodham AW. The role of the lumbar multifidus in chronic low back pain: a review. $P M R$. 2010;2(2):142-146,167. 
79. Hansen L, de Zee M, Rasmussen J, Andersen TB, Wong C, Simonsen EB. Anatomy and biomechanics of the back muscles in the lumbar spine with reference to biomechanical modeling. Spine (Phila Pa 1976). 2006;31(17):1888-1899.

80. Bodine SC, Roy RR, Eldred E, Edgerton VR. Maximal force as a function of anatomical features of motor units in the cat tibialis anterior. J Neurophysiol. 1987;57(6):1730-1745.

81. Sirca A, Kostevc V. The fibre type composition of thoracic and lumbar paravertebral muscles in man. J Anat. 1985;141:131-137.

82. Dickx N, Cagnie B, Achten E, Vandemaele P, Parlevliet T, Danneels L. Differentiation between deep and superficial fibers of the lumbar multifidus by magnetic resonance imaging. Eur Spine J. 2010;19(1):122-128.

83. Johnson M, Polgar J, Weightman D, Appleton D. Data on the distribution of fiber types in thirty six human muscles: an autopsy study. J Neurol Sci. 1973;18(1):111-129.

84. Polgar J, Johnson MA, Weightman D, Appleton D. Data on fibre size in thirty-six human muscles. An autopsy study. J Neurol Sci. 1973;19(3):307-318.

85. Peck D, Buxton D, Nitz A. A comparison of spindle concentrations in large and small muscles acting in parallel combinations. J Morphol. 1984;180(3):243-252.

86. Nitz A, Peck D. Comparison of muscle spindle concentrations in large and small human epaxial muscles acting in parallel combinations. Am Surg. 1986;52(5):273-277.

87. Yamashita T, Minaki Y, Oota I, Yokogushi K, Ishii S. Mechanosensitive afferent units in the lumbar intervertebral disc and adjacent muscle. Spine (Phila Pa 1976). 1993;18(15):2252-2256.

88. Wilke HJ, Wolf S, Claes L, Arand M, Wiesend A. Stability increase of the lumbar spine with different muscle groups. A biomechanical in vitro study. Spine (Phila Pa 1976). 1995;20(2):192-198.

89. Young A. Current issues in arthrogenous inhibition. Ann Rheum Dis. 1993;52(11):829-834.

90. Palmieri RM, Ingersoll CD, Hoffman MA, Cordova ML, Porter DA, Edwards JE, et al. Arthrogenic muscle response to a simulated ankle joint effusion. Br J Sports Med. 2004;38(1):26-30.

91. Felder A, Ward SR, Lieber RL. Sarcomere length measurement permits high resolution normalization of muscle fiber length in architectural studies. J Exp Biol. 2005;208(Pt 17):3275-3279.

92. Ahern DK, Follick MJ, Council JR, Laser-Wolston N, Litchman H. Comparison of lumbar paravertebral EMG patterns in chronic low back pain patients and non-patient controls. Pain. 1988;34(2):153-160.

93. Bohannon RW, Larkin PA. Passive ankle dorsiflexion increases in patients after a regimen of tilt table-wedge board standing. A clinical report. Phys Ther. 1985;65(11):1676-1678.

94. Magnusson SP, Simonsen EB, Aagaard P, Moritz U, Kjaer M. Contraction specific changes in passive torque in human skeletal muscle. Acta Physiol Scand. 1995;155(4):377-386.

95. Bunderson NE, Burkholder TJ, Ting LH. Reduction of neuromuscular redundancy for postural force generation using an intrinsic stability criterion. J Biomech. 2008;41(7):1537-1544.

96. Masi AT, Sierakowski S, Kim JM. Jacques Forestier's vanished bowstring sign in ankylosing spondylitis: a call to test its validity and possible relation to spinal myofascial hypertonicity. Clin Exp Rheumatol. 2005;23(6):760-766.

97. Hooper AC. Length, diameter and number of ageing skeletal muscle fibres. Gerontology. 1981;27(3):121-126.

98. Larsson L. Morphological and functional characteristics of the ageing skeletal muscle in man. A cross-sectional study. Acta Physiol Scand Suppl. 1978;457:1-36.

99. Alnaqeeb MA, Al Zaid NS, Goldspink G. Connective tissue changes and physical properties of developing and ageing skeletal muscle. J Anat. 1984;139(Pt 4):677-689.

100. Gajdosik RL, Vander Linden DW, Williams AK. Concentric isokinetic torque characteristics of the calf muscles of active women aged 20 to 84 years. J Orthop Sports Phys Ther. 1999;29(3):181-190.

101. Booth FW. Physiologic and biochemical effects of immobilization on muscle. Clin Orthop Relat Res. 1987;(219):15-20.

102. Appell HJ. Muscular atrophy following immobilization. A review. Sports Med. 1990;10(1):42-58.

103. Rissanen A, Kalimo H, Alaranta H. Effect of intensive training on the isokinetic strength and structure of lumbar muscles in patients with chronic low back pain. Spine (Phila Pa 1976). 1995;20(3):333-340.

104. Mueller M, Breil FA, Vogt M, Steiner R, Lippuner K, Popp A, et al. Different response to eccentric and concentric training in older men and women. Eur J Appl Physiol. 2009;107(2):145-153.

105. Blazevich AJ. Effects of physical training and detraining, immobilization, growth and aging on human fascicle geometry. Sports Med. 2006;36(12):1003-1017.

106. Hides JA, Stokes MJ, Saide M, Jull GA, Cooper DH. Evidence of lumbar multifidus muscle wasting ipsilateral to symptoms in patients with acute/subacute low back pain. Spine (Phila Pa 1976). 1994;19(2):165-172.

107. Hides JA, Richardson CA, Jull GA. Multifidus muscle recovery is not automatic after resolution of acute, first-episode low back pain. Spine (Phila Pa 1976). 1996;21(23):2763-2769.

108. Hides JA, Jull G, Richardson C. Long-term effects of specific stabilizing exercises for first-episode low back pain. Spine (Phila Pa 1976). 2001;26(11):E243-E248.

109. Hides JA, Stanton WR, McMahon S, Sims K, Richardson CA. Effect of stabilization training on multifidus muscle crosssectional area among young elite cricketers with low back pain. J Orthop Sports Phys Ther. 2008;38(3):101-108.

110. MacDonald DA, Moseley GL, Hodges PW. The lumbar multifidus: does the evidence support clinical beliefs? Man Ther. 2006;11(4):254-263.

111. Gordon TP, Sage MR, Bertouch JV, Brooks PM. Computed tomography of paraspinal musculature in ankylosing spondylitis. J Rheumatol. 1984;11(6):794-797.

112. Schleip R, Klingler W, Zorn A. Biomechanical properties of fascial tissues and their role as pain generators. J Musculoskelet Pain. 2010;:[in press].

113. Harmon O, Dietrich MR, eds. Rebels, Mavericks, and Heretics in Biology. New Haven, CT: Yale University Press; 2009.

114. Flake GW. The Computational Beauty of Nature. Cambridge, MA: The MIT Press; 1998.

Corresponding author: Alfonse T. Masi, Professor of Medicine and Epidemiology, University of Illinois College of Medicine at Peoria (UICOMP), One Illini Drive, Peoria, Illinois 61656 USA.

E-mail: amasi@uic.edu 